\title{
Reduced Brainstem Inhibition during Anticipated Pelvic Visceral Pain Correlates with Enhanced Brain Response to the Visceral Stimulus in Women with Irritable Bowel Syndrome
}

\author{
Steven M. Berman, Bruce D. Naliboff, Brandall Suyenobu, Jennifer S. Labus, Jean Stains, Gordon Ohning, \\ Lisa Kilpatrick, Joshua A. Bueller, Kim Ruby, Johanna Jarcho, and Emeran A. Mayer \\ Departments of Medicine, Physiology, Psychiatry and Biobehavioral Sciences, and Brain Research Institute, University of California, Los Angeles Center for \\ Neurovisceral Sciences and Women's Health, David Geffen School of Medicine at University of California, Los Angeles, Los Angeles, CA 90073, and \\ Veterans Affairs Greater Los Angeles Healthcare System, Los Angeles, California 90073
}

\begin{abstract}
Cognitive factors such as fear of pain and symptom-related anxiety play an important role in chronic pain states. The current study sought to characterize abnormalities in preparatory brain response before aversive pelvic visceral distention in irritable bowel syndrome (IBS) patients and their possible relationship to the consequences of distention. The brain functional magnetic resonance imaging (fMRI) blood oxygen level-dependent (BOLD) response to anticipated and delivered mild and moderate rectal distention was recorded from 14 female IBS patients and 12 healthy controls. During cued anticipation of distention, activity decreased in the insula, supragenual anterior cingulate cortex (sACC), amygdala, and dorsal brainstem (DBS) of controls. IBS patients showed less anticipatory inactivation. Group differences were significant in the right posterior insula and bilateral DBS. Self-rated measures of negative affect during scanning were higher in patients than controls $(p<0.001)$, and the anticipatory BOLD decreases in DBS were inversely correlated with these ratings. During subsequent distention, both groups showed activity increases in insula, dorsal ACC, and DBS and decreases in the infragenual ACC. The increases were more extensive in patients, producing significant group differences in dorsal ACC and DBS. The amplitude of the anticipatory decrease in the pontine portion of DBS was associated with greater activation during distention in right orbitofrontal cortex and bilateral sACC. Both regions have been associated previously with corticolimbic inhibition and cognitive coping. Deficits in preparatory inhibition of DBS, including the locus ceruleus complex and parabrachial nuclei, may interfere with descending corticolimbic inhibition and contribute to enhanced brain responsiveness and perceptual sensitivity to visceral stimuli in IBS.
\end{abstract}

Key words: fMRI; brainstem; irritable bowel syndrome; anticipation; pain; corticolimbic inhibition

\section{Introduction}

Irritable bowel syndrome (IBS) is one of the most common chronic functional pain syndromes, which also include fibromyalgia and chronic pelvic pain (Diatchenko et al., 2006; Drossman, 2006). These disorders frequently overlap and share many epidemiological, clinical, and pathophysiological features (Wessely et al., 1999; Keogh et al., 2003), including symptom-related anxiety (Asmundson et al., 1999; Mayer et al., 2001; Goubert et al., 2004; Labus et al., 2007) and central pain amplification (Gracely et al.,

Received June 1, 2007; revised 0ct. 3, 2007; accepted 0ct. 20, 2007.

This work was supported by National Institutes of Health Grants DK48351 (E.A.M.), P50DK64539 (E.A.M.), R24 AT002681 (E.A.M.), and NR04881 (B.D.N.) and by Novartis Pharmaceuticals. Preliminary reports were presented at the following annual meetings: American Gastroenterological Association, Los Angeles, CA, May, 2006; Society for Neuroscience, Atlanta, GA, October, 2006. We thank Cathy Lui and Laura Frischer for figure construction and Teresa Olivas for outstanding editorial assistance.

Correspondence should be addressed to Dr. Emeran A. Mayer, Center for Neurovisceral Sciences and Women's Health, Veterans Affairs Greater Los Angeles Healthcare System, Building 115, Room 223, 11301 Wilshire Blvd, Los Angeles, CA 90073. E-mail: emayer@ucla.edu.

DOI:10.1523/JNEUROSCI.2500-07.2008

Copyright $\odot 2008$ Society for Neuroscience $\quad$ 0270-6474/08/280349-11\$15.00/0
2004; Mayer et al., 2006). Symptom-related anxiety is a key predictor of IBS diagnostic status and mediates the relationship between psychological distress and symptom severity (Porro et al., 2002, 2003). Brain mechanisms underlying this relationship are unknown but may involve altered preparation for expected pain (Coull, 1998; Hsieh et al., 1999; Sawamoto et al., 2000; Porro et al., 2004).

Expectation of pain elicits multiple responses, including arousal, cognitive appraisal, memory retrieval, conditioning, and either orienting to or diverting attention away from the expected site of stimulation. In general, coping responses inhibit or amplify the motivational salience of pain. Inhibition minimizes discomfort/distraction when expected pain is tolerable, and competing rewards can be obtained by ignoring it. Amplification enhances arousal and vigilance when expected pain is dangerous (Bushnell et al., 1985), thereby minimizing reaction time and tissue damage. Hypervigilance to aversive stimuli is a hallmark of functional pain disorders, including IBS (Whitehead and Palsson, 1998).

Expectation-related neural activity consistent with increased 
afferent sensitivity has been observed in somatosensory cortex, anterior cingulate cortex (ACC), anterior insula (aINS), thalamus, and spinal dorsal horn (Hsieh et al., 1999; Porro et al., 2002; Porro et al., 2004; Lieberman, 2005). Inhibition of limbic brain activity during anticipation of pain has often been reported in experimental studies where healthy participants are asked to tolerate maximal pain but know they will not sustain serious damage and can terminate pain at will. The brain structures most commonly inhibited are limbic (infragenual/perigenual) portions of the ACC, often with contiguous ventromedial prefrontal cortex (Porro et al., 1998, 2004; Hsieh et al., 1999; Simpson et al., 2001), the functionally connected amygdala (AMYG) (Berman et al., 2002a; Bonaz et al., 2002; Petrovic and Ingvar, 2002; Petrovic et al., 1999, 2001), or both (Derbyshire et al., 1997). An important role of the right ventrolateral prefrontal cortex (vlPFC) or orbitofrontal cortex (OFC) in driving this inhibition of limbic activity has been implicated in imaging studies using experimental techniques, including distraction, hypnosis, expected control, and placebo (for review, see Petrovic and Ingvar, 2002; Mayer et al., 2006).

The perceptual hypersensitivity to potentially aversive gut stimuli characteristic of IBS patients may be associated with deficits in corticolimbic inhibition and increased activation of central arousal circuits during cued delivery of discomfort. We studied brain responses during expectation and delivery of aversive rectal pressure in 26 adult females and hypothesized that IBS patients would show the following: (1) failure to inhibit arousal and limbic brain circuits during expectation, which would correlate with (2) affective stimulus ratings and (3) brain responses to the aversive stimulus.

\section{Materials and Methods}

Subjects. Seventeen adult (acceptable ages were from 18 to 60 years of age) woman with IBS-C, and 15 healthy control women were recruited by fliers, newspaper advertisements, and referrals. Brain images from three control participants and one IBS patient were lost because of equipment failure, and two additional IBS patients were unable to tolerate the experimental procedure. Data from the remaining 14 IBS patients (mean age, 36.1; SD, 8.1) and 12 healthy controls (mean age, 36.3; SD, 7.3) are reported herein. Menstrual cycle phase during functional magnetic resonance imaging (fMRI) testing was estimated from the reported start date of last menses. Two IBS patients and three healthy subjects could not be assessed. Three were postmenopausal or perimenopausal (highly variable with over 6 weeks since last menses), and two provided no information on the start date of last menses. For the remaining subjects, there was a relatively random distribution with approximately equal proportions of women in the follicular and luteal stages (IBS, 4 vs 8 ; control, 4 vs 5 , respectively).

All participants were right handed. On clinical examination by a gastroenterologist, they were clinically and endoscopically without inflammatory or structural intestinal disease. All IBS patients fulfilled Rome II criteria for IBS-C.

Additional exclusionary criteria for either group were abdominal surgery, major medical illness, current or recent history of drug or alcohol abuse, intake of $>4 \mathrm{U}$ of alcohol per day, morbid obesity, metal implants, or large tattoos. All participants were evaluated for depression and anxiety symptoms using the Hospital Anxiety and Depression Scale (HAD) (for review, see Bjelland et al., 2002). Patients with a clinical diagnosis of an anxiety disorder or major depressive episode were excluded. Written and verbal informed consents were obtained from all subjects. The protocol was approved by the University of California, Los Angeles Human Subject Protection Committee.

Symptom assessments. IBS patients filled out a brief IBS symptom questionnaire and daily symptom diaries for 2 weeks preceding fMRI testing. Each diary consisted of 7-point numerical rating scales for abdominal discomfort/pain and for abdominal bloating. The number of bowel movements and presence of either hard or loose/watery stool were also recorded. On the day of MRI testing, IBS patients rated both the intensity and unpleasantness of their gastrointestinal symptoms during the past $24 \mathrm{~h}$ using descriptor anchored visual analog scales (Gracely et al., 1978).

Visceral distention procedure. Distention of the rectum was accomplished using a computer-driven pump (barostat) programmed to deliver phasic pressure steps $(38 \mathrm{ml} / \mathrm{s})$ separated by interinflation intervals at the resting pressure. Methods for balloon insertion and inflation were as described previously (Naliboff et al., 1997). In vitro and in vivo validations of this distention device have been published previously (Lembo et al., 1994; Mertz et al., 1995). All studies were performed after an $8 \mathrm{~h}$ fast and application of 2 Fleet enemas (C.B. Fleet, Lynchburg, VA). Affective and perceptual responses to controlled rectal distention were assessed before the MRI protocol.

Affective ratings. Before and after the visceral distention protocol, all participants rated their current mood using the Stress Symptom Rating scales (SSR). To best capture mood state during the session, the mean of the two assessments was used. The SSR is a validated multidimensional instrument for measuring acute changes in mood during experimental stress (Naliboff et al., 1991). It consists of 12 semantic differential scales, each of which is a $10 \mathrm{~cm}$ line anchored by opposite descriptor words (e.g., calm-stressed). Pairs of scales are averaged to assess subjective mood on dimensions of stress, anger, anxiety, fatigue, arousal, and attentiveness. They have been validated previously in studies of acute psychological stress (Mertz et al., 1995; Dickhaus et al., 2003).

Discomfort threshold during rectal distention. Discomfort thresholds for rectal distention were assessed at the beginning of the MRI session, and ratings of the highest intensity and discomfort associated with any rectal distention were assessed at the conclusion. A sensory tracking protocol was used to determine discomfort thresholds. Details of the threshold tracking have been reported previously (Naliboff et al., 1997). Briefly, the electronic distention device was programmed to deliver intermittent phasic stimuli (30 s duration; $5 \mathrm{mmHg}$ increments) separated by an interpulse interval (30 s duration; $5 \mathrm{mmHg}$ ) within a stimulus tracking paradigm (15 distention trials). During each stimulus and rest, subjects were prompted by the distention device to report the intensity of their sensation on a 4-point scale. If the sensation indicated by the subject was below the discomfort level (i.e., no sensation or moderate), the next stimulus was randomized to either stay the same or increase by $5 \mathrm{mmHg}$ to a maximum value of $45 \mathrm{mmHg}$. If the sensation indicated by the subject was discomfort or pain, the next stimulus was randomized to stay the same or to decrease by $5 \mathrm{mmHg}$. The discomfort threshold was calculated as the average of the last six pressures given (Naliboff et al., 1997).

MRI protocol. The subject was positioned in the MRI scanner on a cushioned headrest. The subject was given earplugs to reduce scanner noise and was fitted with an earphone headset with microphone to allow two-way communication with the experimenters during scanning. A mirrored prism viewing assembly allowed the subject to see the inflation and response cues, which were presented on a notebook computer placed against the viewing window within the scanner control room. Tape was put across the forehead, and padding inserts were used to restrict movement.

The subject was given the following instructions: "the computer will now begin to inflate the balloon to various pressures in no particular order. You will probably feel sensations of varying intensity. At the beginning and end of the inflation there will be a visual cue. Remember, when you see the (blue/white) rectangle, the inflation will soon begin, and when you see the color change, you will need to rate the inflation. Indicate the intensity of your sensation by lifting a closed fist if you felt LOW or NO INTENSITY, lifting one finger for a MIDDLE INTENSITY, or two fingers for a HIGH level of INTENSITY. Please answer immediately when the visual cue comes on. In the case that you can no longer tolerate the test, tell us immediately, but please try to finish the test. Remember that during this part of the test, the intensity of the inflations will not be in any particular order."

Four to six 10 min stimulus sets containing 16 inflation trials each were administered. The first trial in each set always used a pressure of 45 $\mathrm{mmHg}$. This was followed by five additional $45 \mathrm{mmHg}$ inflations, five 25 


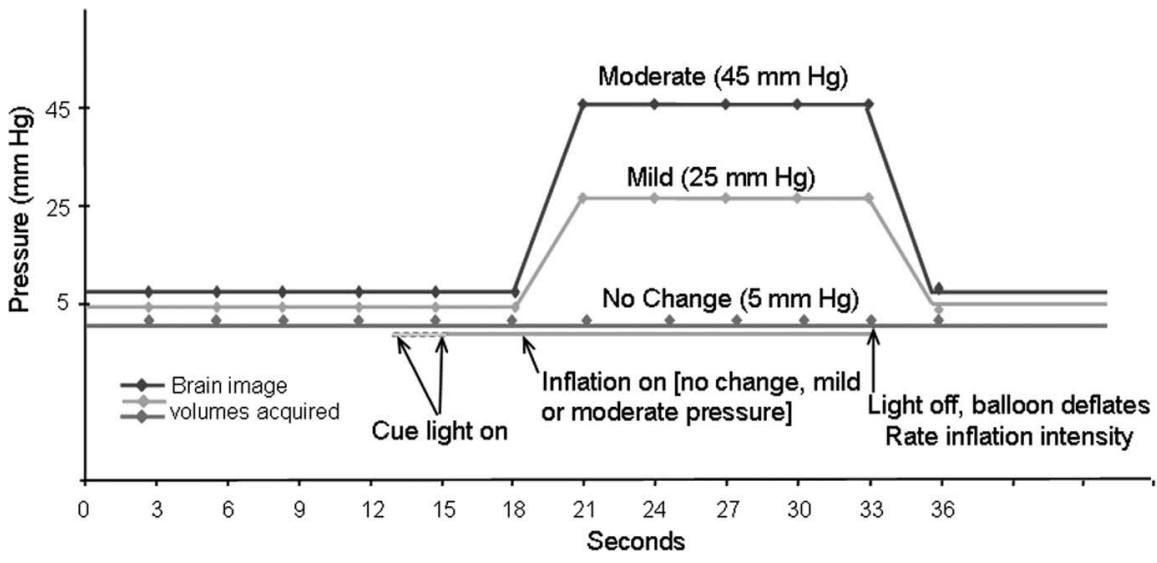

Figure 1. Trial design. In each session, 20-30 moderate intensity rectal distentions ( $15 \mathrm{~s}$ at $45 \mathrm{mmHg}$ ) were randomly intermingled with an equal number of mild distentions $(25 \mathrm{mmHg})$ and sham distentions $(5 \mathrm{mmHg})$.

$\mathrm{mmHg}$ inflations, and five trials at the baseline pressure of $5 \mathrm{mmHg}$ (sham inflation) in pseudorandom order.

Each trial consisted of $18 \mathrm{~s}$ before balloon inflation, followed by $15 \mathrm{~s}$ of inflation at the designated pressure and $3 \mathrm{~s}$ for deflation and rating (Fig. 1). The visual cue preceded the first inflation by $2 \mathrm{~s}$ and the other 15 inflations by 3,4 , or $5 \mathrm{~s}$ (rectangular distribution). The cue was removed at the end of the inflation period. This signaled the end of a trial, at which point subjects rated the intensity of the stimulus. Ratings were made on a simple three-point scale. In contrast to the earlier discomfort threshold determination (see above), these ratings were not intended as a psychophysical measure. They were designed to keep subjects focused on the stimuli and provided a validity check of discrimination between stimulus levels. There was a brief rest $(<1 \mathrm{~min})$ between sets. After the last set, a structural T1-weighted whole brain image was acquired (see below).

All participants then rated the intensity and unpleasantness of the highest level of visceral inflation experienced during the session using visual analog scales (Gracely et al., 1978) and completed a final assessment of current mood (SSR). The catheter was removed, and the subject was excused.

MRI acquisition. Brain images were acquired on a 1.5T MRI scanner (Siemens Sonata; Siemens, Erlangen, Germany). First, a sagittal scout was used to position the head. Then, functional $\mathrm{T} 2{ }^{\star}$ weighted gradientrecalled echo-planar images with blood oxygen level-dependent (BOLD) contrast (repetition time, $2970 \mathrm{~ms}$; echo time, $42 \mathrm{~ms}$; flip angle, $80^{\circ}$; slice thickness, $4 \mathrm{~mm}$ with a $1 \mathrm{~mm}$ interslice interval; matrix, $64 \times 64$; in-plane resolution, $3.12 \mathrm{~mm}^{2}$ ) were acquired during each $10 \mathrm{~min}$ stimulus set and constituted an fMRI run. After discarding the first two volumes of each 10 min run, 25 axial slices were recorded for each of 200 functional whole-brain volumes and saved to disk for off-line analysis. After the functional runs were completed, a magnetization-prepared rapidacquisition gradient echo pulse sequence was used to acquire a threedimensional structural T1-weighted image (resolution, $1.0 \times 1.0 \times 1.0$ $\mathrm{mm}$ ) that covered the entire brain.

Data analysis. BOLD fMRI images from each session were first examined by a software program that processes a time series of images and identifies those likely to be artifacts based on outliers in motion, signal intensity, and other sources of change between successive images (Outlier; provided by M. Cohen, University of California, Los Angeles, Los Angeles, CA). The signal spike threshold used by the Outlier program was $30 \%$ above background noise levels determined on a per-slice basis. The motion threshold was $2 \%$. Images tagged as outliers were visually inspected, and unacceptable images, generally resulting from intensity spikes in a single slice, were replaced by means of the adjacent images. The following preprocessing procedures were then implemented using statistical parametric mapping 2 (SPM2) (Wellcome Department of Cognitive Neurology, London, UK; http://www.fil.ion.ucl.ac.uk/spm/ software/spm 2/). Correction for acceptable head movement between the images in each session was performed by alignment with one image. Each subject's realigned images were resliced to isotropic $2 \mathrm{~mm}^{3}$ voxels and normalized by linear and nonlinear transformations into a standardized anatomical space [Montreal Neurological Institute (MNI) space: an average of 152 whole-brain T1 images collected at the Montreal Neurological Institute] The $x, y, z$ coordinates of MNI space represent approximate millimeter distances from the anterior commissure (voxel, 000 ) with positive values to the right, anterior, and superior directions respectively. After normalization, a $5 \mathrm{~mm}$ (full width half maximum) Gaussian filter was applied to each image.

$S P M$. The images associated with the initial trial of each stimulus set were discarded, both to reduce novelty effects, and because the first trial always used the same pressure and therefore may have become predictable across sets. The remaining images were assigned to six conditions (Fig. 1): (1) rest (five images per trial modeled as an implicit baseline), (2) cue (one image per trial), (3) mild distention (five images on one-third of the trials), (4) moderate distention (five images on one-third of the trials), (5) sham distention (five images on one-third of the trials), and (6) rate (one image per trial). Conditions 2-5 were compared with the implicit baseline (rest) in individual fixed-effects linear contrasts for each subject.

SPM2 treats each voxel according to a general linear model, taking into account the intrinsic autocorrelation imposed by the hemodynamic response. For each condition, activated and deactivated voxels were identified using an $\alpha$ level of $p<0.01$ (uncorrected for multiple comparisons) and used to construct individual statistical parametric maps. All suprathreshold voxels in a statistical parametric map are partitioned into clusters of contiguous (touching) voxels. SPM2 calculates probability values for the spatial extent of each cluster (size in voxels) and the strength of the effect at each individual voxel. The multiple comparisons problem is addressed using continuous random field theory, assuming the statistic image to be a good lattice representation of an underlying continuous stationary random field. This results in inference based on corrected $p$ values.

A region-of-interest (ROI) approach used the SPM2 small volume correction procedure to separately quantify BOLD signal increases and decreases within seven a priori brain areas previously associated with pain or functional response to visceral distention. ROIs were drawn by the first author on the structural MR template to which all scans were normalized using MEDx Software (Sensor Systems, Sterling, VA). The name of each ROI is followed by the abbreviation and size (number of voxels) as follows: anterior insula (aINS/980), posterior insula (pINS/ 1269), posterodorsal anterior cingulate (dACC/512), supragenual anterior cingulate (sACC/877), infragenual anterior cingulate (iACC/357), amygdala (AMYG/246), and dorsal brainstem (DBS/351).

The DBS region contains multiple small structures implicated in the modulation of pain, including the medial raphe (dorsal, median, and paramedian nuclei), the locus ceruleus complex (LCC), and laterally adjacent parabrachial nuclei (PBN) in the pons and in the midbrain the periaqueductal gray (PAG) and nucleus cuneiforme (NCF). The latter has recently been associated specifically with visceral pain processing (Dunckley et al., 2005). We refer to the pontine portion of the DBS as the LCC-PBN complex and to the midbrain portion of the DBS as the PAGNCF complex. These subdivisions represent the approximate spatial resolution limit of the functional images.

Within each structure, hemisphere, and condition, the voxel t-scores associated with the peak BOLD increase and decrease were corrected for the volume of the ROI using the SPM2 family-wise error algorithm. If a corrected probability value was less than or equal to 0.05 , the ROI was considered to contain a significant activation or deactivation. Using this method, the same ROI can contain both activations and deactivations (although not at the same voxel).

Although formal hypothesis testing used the peak effect criterion presented above, a secondary form of evidence within each ROI quantified 
the probability associated with the spatial extent (number of voxels; i.e., size) of the cluster of contiguous voxels (i.e., the set of adjacent voxels that pass the statistical threshold) containing the peak effect. This is a conservative use of the spatial extent criterion, because the voxel of peak effect size may not be in the largest cluster within an ROI. Spatial extent evidence generally correlates well with peak voxel evidence but can be more sensitive to weak but spatially extended effects. The proportion of the ROI that passed the uncorrected $p<0.01$ threshold was also noted, although we know of no accepted statistical test for this measure.

Individual subject contrast images were generated for each stimulus (cue, $5 \mathrm{~mm}$ sham inflation, $25 \mathrm{~mm}$ inflation, $45 \mathrm{~mm}$ inflation) and entered into second level random effects SPM analyses. These quantified BOLD changes in female IBS-C patients $(n=14)$, healthy female controls $(n=12)$, and differences between the two groups. This represents a traditional approach for assessing brain images that have been normalized into the same atlas space (see preprocessing above) combining data across subjects at each voxel before evaluating evidence of BOLD increases and/or decreases within each structure.

The 26 subject contrast images for the cue were also entered into two types of covariate analyses. First, we assessed the relationship of the cue-elicited BOLD response to the visceral discomfort threshold recorded at the beginning of the session. Next, we assessed the relationship of the cue-elicited BOLD response to the SSR negative affect mood measures.

Finally, we assessed the relationship of the cue-elicited BOLD signal at the voxel of maximal group difference in the right DBS (voxel, $4,-30$, -24 in LCC-PBN) and left DBS (voxel, $-4,-26,-12$ in PAG-NCF) to the BOLD response during $45 \mathrm{~mm} \mathrm{Hg}$ distention. Because that analysis had implications for preparation of coping responses, an additional ROI was assessed in the right OFC, which has been implicated previously in executive coping (Leuchter et al., 2002; Petrovic and Ingvar, 2002; Petrovic et al., 2002, 2005; Lieberman et al., 2004). For comparative purposes, its left hemisphere homolog was also examined.

\section{Results}

\section{Characteristics of the two groups and behavioral responses}

Table 1 summarizes the ages and HAD scores in both groups and measures of IBS symptom severity in the IBS-C group. All patients had IBS symptoms for at least 3 years. On a five-point scale, they rated their usual symptoms as moderate (3), severe (4), or very severe (5), with an average rating between moderate and severe (mean/SD, 3.5/0.76). All depression and anxiety scores were within the normal range for all healthy controls and for 12 of 14 patients. As a group, patients reported more anxiety and depression than healthy controls (both $p<0.001$ ), and this was true even if the two patients who had clinically significant anxiety scores were excluded. Table 2 presents self report measures of mood and stimulus perception during the MRI session.

\section{Ratings of affective state}

Patients robustly reported more stress, anxiety, and anger than healthy controls both before and after the rectal distention protocol (Table 2). Moreover, these three measures were highly intercorrelated (Pearson product-moment coefficients ranged from 0.84 to 0.93 ), suggesting they can be conceptualized as rep- resenting a higher-order factor of negative affect. IBS patients also reported more fatigue, but the effect was less robust and did not correlate with the other measures.

\section{Perceptual stimulus ratings}

Rectal distention discomfort thresholds measured by the sensory tracking protocol were significantly lower in IBS-C patients compared with healthy controls (Table 2). However, the groups did not differ in the intensity and discomfort associated with the maximal distention pressure experienced during the session.

\section{Behavioral responses during fMRI scanning}

Both groups discriminated well between the three levels of rectal distention pressure. Subjects rated the sham condition (no change from resting pressure of $5 \mathrm{mmHg}$ ), reliably the lowest on the three-scale from 1 to 3 (mean \pm SD: IBS-C, $1.22 \pm 0.38$; control, $1.05 \pm 0.17$ ). The ratings for the $25 \mathrm{mmHg}$ pressure were intermediate (IBS-C, $2.13 \pm 0.53$; control, $1.78 \pm 0.38$ ), and the ratings for the $45 \mathrm{mmHg}$ pressure were highest (IBS-C, $2.83 \pm$ 0.32; control, $2.78 \pm 0.38$ ). In all subjects, the mean rating for the $25 \mathrm{mmHg}$ pressure was between the mean rating for the 5 and 45 mmHg pressure.

\section{Brain responses during $\mathrm{fMRI}$ scanning}

ROI results for each group and a group comparison are presented in Figure 2 and Table 3.

\section{Expectation condition (cue light)}

As a group, controls, but not patients, showed widespread BOLD decreases in regions of the homeostatic afferent processing network, including all tested ROIs with the exception of bilateral 


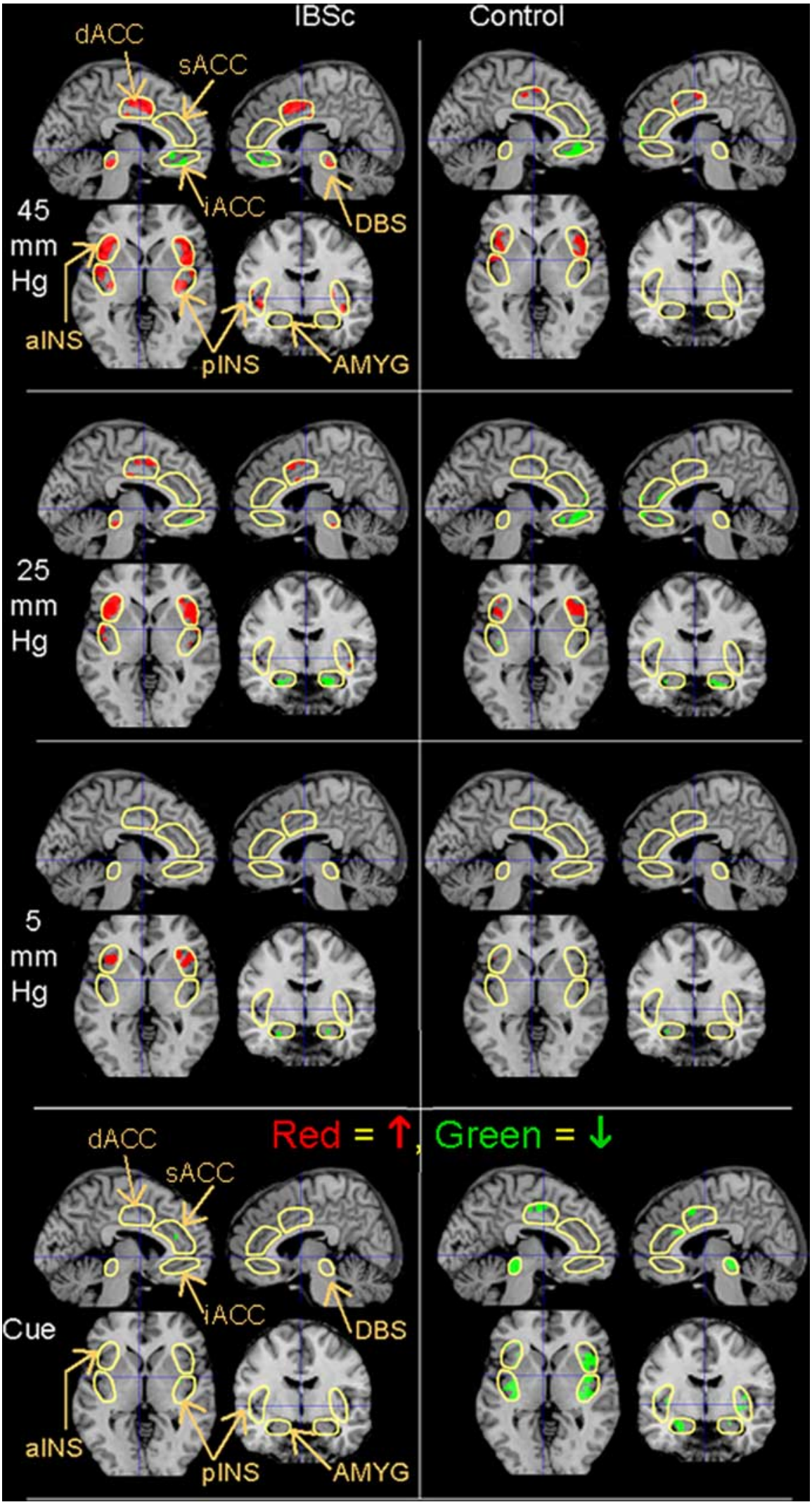

Figure 2. Group functional activation maps. BOLD responses $(p<0.01)$ within the ROIs (outlined in yellow) in female IBS-C patients and healthy controls in response to the cue light and the subsequent 5 (sham), 25 , and $45 \mathrm{mmHg}$ distentions are superimposed on a structural MRI representative of MNI space for a sagittal slice $5 \mathrm{~mm}$ from the midline in each hemisphere (left side, left hemisphere), a coronal slice $6 \mathrm{~mm}$ behind the anterior commissure, and a transaxial slice through the anterior commissure. All figures depict neurological orientation (left $=$ left).
iACC and left dACC (Mayer et al., 2006). Neither group showed a significant BOLD increase during the cue condition. This produced significant group differences in the right pINS and bilateral DBS.

Because we had not selected the thalamus, another region of the homeostatic afferent processing network, as an a priori region, we conducted a post hoc examination of activity during the expectation condition within right and left thalamus, operationalized as $10 \mathrm{~mm}$ radius spheres centered at MNI $\pm 10,-18,10$. Healthy subjects showed extensive deactivation during the cue, both by the criterion of spatial extent [left (L), 51\%, $p<.0005 ; r=$ $42 \%, p=0.001$ ] and voxel height [L: $t=$ 5.11, $p=0.006$; right $(\mathrm{R}): t=4.30$, $p=0.032]$. In contrast, there was no spatial extent evidence for cue-related deactivation in the patients ( $\mathrm{L}, 8 \% ; \mathrm{R}, 3 \%$; NS). However, patients did show cue-related deactivation for a voxel in the pulvinar nucleus of the left thalamus $(t=5.15 ; p=$ 0.006).

\section{$45 \mathrm{mmHg}$ distention}

IBS patients showed bilateral BOLD increases in the four homeostatic afferent processing network ROIs (aINS, pINS, $\mathrm{dACC}$, and DBS). The iACC showed bilateral BOLD decrease. No significant effects were seen in the AMYG.

In contrast, controls demonstrated significant BOLD increases only in bilateral INS (anterior and posterior) and BOLD decrease in left iACC. Group comparison indicated greater BOLD increase in patients within the left DBS, with spatial extent evidence suggesting this may also be true within the left dACC (Table 3 ).

\section{$25 \mathrm{mmHg}$ distention}

In IBS patients, regions where $45 \mathrm{mmHg}$ distention produced a BOLD increase were also increased during the $25 \mathrm{mmHg}$ distention (bilateral aINS, pINS, dACC, and DBS), but the effects were attenuated in both cluster size and maximum t-score. There were BOLD decreases in right iACC and bilateral AMYG.

Controls demonstrated significant BOLD increases only in bilateral aINS. They had BOLD decreases in left iACC and right AMYG. Group comparison yielded suggestive trends $(p<0.07)$ for greater BOLD increases in the patients within right pINS and right dACC.

\section{Sham distention $(5 \mathrm{mmHg}$ )}

IBS patients generated significant BOLD increases during sham distention only in 
Table 3. BOLD responses to experimental stimuli

\begin{tabular}{|c|c|c|c|c|c|c|c|c|c|c|c|c|c|c|c|c|c|c|c|}
\hline & & \multicolumn{6}{|l|}{ IBS } & \multicolumn{6}{|c|}{ Control } & \multicolumn{6}{|c|}{ IBS > control } \\
\hline & & \multicolumn{3}{|c|}{ Cluster } & \multicolumn{3}{|l|}{ Voxel } & \multicolumn{3}{|c|}{ Cluster } & \multicolumn{3}{|l|}{ Voxel } & \multicolumn{3}{|c|}{ Cluster } & \multicolumn{3}{|l|}{ Voxel } \\
\hline & & $k$ & $p$ (cor) & $\mathrm{ROI}$ & $t$ & $p$ (cor) & $x, y, z$ & $k$ & $p$ (cor) & $\mathrm{ROI}^{0.01}$ & $t$ & $p$ (cor) & $x, y, z$ & $k$ & $p$ (cor) & $\mathrm{ROI} \mathrm{I}^{0.01}$ & $t$ & $p$ (cor) & $x, y, z$ \\
\hline \multicolumn{20}{|l|}{$45 \mathrm{mmHg}$ increase } \\
\hline \multirow[t]{2}{*}{ Anterior insula } & $\mathrm{L}$ & 620 & $0.00^{*}$ & $69 \%$ & 7.61 & $0.00^{*}$ & $-36,26,-8$ & 264 & 0.001 & $29 \%$ & 5.63 & 0.002 & $-40,8,6$ & & & & & & \\
\hline & $\mathrm{R}$ & 540 & $0.00^{*}$ & $51 \%$ & 6.16 & 0.001 & $38,24,-12$ & 279 & 0.001 & $29 \%$ & 4.78 & 0.02 & $42,4,2$ & & & & & & \\
\hline \multirow[t]{2}{*}{ Posterior insula } & $\mathrm{L}$ & 371 & $0.00^{*}$ & $32 \%$ & 6.78 & $0.00^{*}$ & $-48,2,2$ & 129 & 0.02 & $9 \%$ & 5.65 & 0.004 & $-40,6,6$ & & & & & & \\
\hline & $\mathrm{R}$ & 320 & $0.00^{*}$ & $30 \%$ & 6.05 & 0.001 & $48,4,0$ & 37 & 0.14 & $4 \%$ & 5.41 & 0.004 & $42,4,4$ & & & & & & \\
\hline \multirow[t]{2}{*}{ Dorsal brainstem } & $\mathrm{L}$ & 130 & 0.005 & $38 \%$ & 5.71 & 0.001 & $-6,-42,-24$ & & & & & & & 10 & 0.17 & $6 \%$ & 3.78 & 0.04 & $-6,-42,-24$ \\
\hline & $\mathrm{R}$ & 150 & 0.003 & $44 \%$ & 6.04 & $0.00^{*}$ & $8,-30,-20$ & & & & & & & & & & & & \\
\hline ACC dorsal & $\mathrm{L}$ & 234 & 0.001 & $43 \%$ & 5.35 & 0.003 & $-8,4,42$ & & & & & & & 51 & 0.05 & $10 \%$ & 3.18 & 0.17 & $-8,12,42$ \\
\hline & $\mathrm{R}$ & 161 & 0.003 & $45 \%$ & 5.15 & 0.003 & $0,16,38$ & & & & & & & & & & & & \\
\hline $45 \mathrm{mmHg}$ decrease & & & & & & & & & & & & & & & & & & & \\
\hline ACC supragenual & $\mathrm{L}$ & & & & & & & & & & & & & & & & & & \\
\hline & $\mathrm{R}$ & 29 & 0.14 & $3 \%$ & 3.74 & 0.09 & $6,34,2$ & & & & & & & & & & & & \\
\hline ACC infragenual & $\mathrm{L}$ & 142 & 0.004 & $39 \%$ & 3.92 & 0.04 & $-6,32,-6$ & 116 & 0.008 & $32 \%$ & 3.74 & 0.05 & $-6,44,-4$ & & & & & & \\
\hline & $\mathrm{R}$ & 12 & 0.16 & $16 \%$ & 3.77 & 0.04 & $10,52,-4$ & & & & & & & & & & & & \\
\hline $25 \mathrm{mmHg}$ increase & & & & & & & & & & & & & & & & & & & \\
\hline Anterior insula & $\mathrm{L}$ & 545 & $0.00^{*}$ & $61 \%$ & 6.14 & 0.001 & $-38,20,-2$ & 245 & 0.002 & $28 \%$ & 5.05 & 0.007 & $-32,28,-6$ & & & & & & \\
\hline & $\mathrm{R}$ & 526 & $0.00^{*}$ & $50 \%$ & 5.81 & 0.002 & $48,10,-2$ & 328 & $0.00^{*}$ & $31 \%$ & 5.99 & 0.001 & $36,22,4$ & & & & & & \\
\hline Posterior insula & $\mathrm{L}$ & 211 & 0.004 & $15 \%$ & 5.64 & 0.003 & $-48,2,0$ & & & & & & & & & & & & \\
\hline & $\mathrm{R}$ & 41 & 0.13 & $6 \%$ & 4.84 & 0.01 & $46,12,-6$ & & & & & & & 28 & 0.19 & $4 \%$ & 4.04 & 0.06 & $40,-14,4$ \\
\hline Dorsal brainstem & $\mathrm{L}$ & 18 & 0.12 & $7 \%$ & 3.65 & 0.05 & $-4,-28,-14$ & & & & & & & & & & & & \\
\hline & $\mathrm{R}$ & 53 & 0.04 & $15 \%$ & 4.34 & 0.01 & $6,-30,-20$ & & & & & & & & & & & & \\
\hline ACC dorsal & $\mathrm{L}$ & 56 & 0.05 & $14 \%$ & 3.87 & 0.05 & $-6,18,42$ & & & & & & & & & & & & \\
\hline & $\mathrm{R}$ & 25 & 0.11 & $14 \%$ & 4.12 & 0.02 & $6,8,28$ & & & & & & & 8 & 0.21 & $2 \%$ & 3.56 & 0.07 & $6,10,28$ \\
\hline $25 \mathrm{mmHg}$ decrease & & & & & & & & & & & & & & & & & & & \\
\hline ACC infragenual & $\mathrm{L}$ & 42 & 0.06 & $12 \%$ & 3.23 & 0.12 & $-8,44,-12$ & 128 & 0.008 & $35 \%$ & 3.66 & 0.05 & $-12,36,-10$ & & & & & & \\
\hline & $\mathrm{R}$ & 53 & 0.04 & $15 \%$ & 5.34 & 0.002 & $12,50,-6$ & 26 & 0.09 & $10 \%$ & 2.97 & 0.16 & $6,52,-4$ & & & & & & \\
\hline Amygdala & $\mathrm{L}$ & 72 & 0.02 & $30 \%$ & 4.39 & 0.01 & $-20,-8,-24$ & 6 & 0.19 & $3 \%$ & 3.43 & 0.07 & $-28,-12,-14$ & & & & & & \\
\hline & $\mathrm{R}$ & 52 & 0.04 & $21 \%$ & 5.37 & 0.001 & $20,-6,-22$ & 31 & 0.07 & $13 \%$ & 3.75 & 0.04 & $20,-6,-22$ & & & & & & \\
\hline $5 \mathrm{mmHg}$ increase & & & & & & & & & & & & & & & & & & & \\
\hline Anterior insula & $\mathrm{L}$ & 201 & 0.004 & $23 \%$ & 5.04 & 0.007 & $-34,22,4$ & & & & & & & & & & & & \\
\hline & $\mathrm{R}$ & 252 & 0.002 & $24 \%$ & 4.39 & 0.03 & $28,26,-2$ & & & & & & & & & & & & \\
\hline ACC dorsal & $\mathrm{L}$ & 14 & 0.18 & $5 \%$ & 3.71 & 0.06 & $-14,0,38$ & & & & & & & & & & & & \\
\hline & $\mathrm{R}$ & & & & & & & & & & & & & & & & & & \\
\hline $5 \mathrm{mmHg}$ decrease & & & & & & & & & & & & & & & & & & & \\
\hline Amygdala & $\mathrm{L}$ & 20 & 0.10 & $8 \%$ & 4.46 & 0.009 & $-26,-4,-24$ & & & & & & & & & & & & \\
\hline & $\mathrm{R}$ & 5 & 0.19 & $2 \%$ & 3.22 & 0.09 & $22,-6,-22$ & & & & & & & & & & & & \\
\hline Cue decrease & & & & & & & & & & & & & & & & & & & \\
\hline Anterior insula & $\mathrm{L}$ & & & & & & & 3 & 0.46 & $11 \%$ & 4.74 & 0.02 & $-28,32,-2$ & & & & & & \\
\hline & $\mathrm{R}$ & & & & & & & 285 & $0.00^{*}$ & $27 \%$ & 4.93 & 0.01 & $36,8,2$ & & & & & & \\
\hline Posterior insula & $\mathrm{L}$ & & & & & & & 218 & 0.002 & $22 \%$ & 4.96 & 0.02 & $-46,0,8$ & & & & & & \\
\hline & $\mathrm{R}$ & & & & & & & 306 & $0.00^{*}$ & $28 \%$ & 5.11 & 0.009 & $42,-14,-6$ & 94 & 0.02 & $9 \%$ & 4.33 & 0.04 & $40,-10,6$ \\
\hline Dorsal brainstem & $\mathrm{L}$ & & & & & & & 198 & 0.001 & $58 \%$ & 4.49 & 0.01 & $-6,-26,-12$ & 85 & 0.01 & $25 \%$ & 4.01 & 0.03 & $-4,-26,-12$ \\
\hline & $\mathrm{R}$ & & & & & & & 118 & 0.005 & $34 \%$ & 4.56 & 0.01 & $10,-24,-6$ & 22 & 0.10 & $9 \%$ & 3.76 & 0.05 & $4,-30,-24$ \\
\hline ACC dorsal & $\mathrm{L}$ & & & & & & & & & & & & & & & & & & \\
\hline & $\mathrm{R}$ & & & & & & & 31 & 0.08 & $7 \%$ & 3.79 & 0.05 & $4,14,42$ & & & & & & \\
\hline ACC supragenual & $\mathrm{L}$ & & & & & & & 68 & 0.04 & $8 \%$ & 3.87 & 0.08 & $-10,32,22$ & & & & & & \\
\hline & $\mathrm{R}$ & & & & & & & 59 & 0.05 & $7 \%$ & 3.62 & 0.13 & $2,30,24$ & & & & & & \\
\hline Amygdala & $\mathrm{L}$ & & & & & & & 98 & 0.008 & $40 \%$ & 4.24 & 0.02 & $-22,-4,-24$ & & & & & & \\
\hline & $\mathrm{R}$ & & & & & & & 9 & 0.16 & $4 \%$ & 3.89 & 0.09 & $26,2,-26$ & & & & & & \\
\hline
\end{tabular}

For each region where peak voxel height or the spatial extent volume-corrected $p<0.1$ of that cluster, the cluster size (number of voxels) is labeled $k$, followed by the volume-corrected probability associated with finding a cluster this large or larger $[p($ cor $)]$. Because the peak cluster is not always within the largest cluster, the two cluster columns are followed by an additional measure of spatial extent, the total percentage of R0l voxels where $p<0.01$ (uncorrected), although there is no accepted statistical test associated with this measure. The three voxel columns present the peak voxel $t$-score, the volume corrected probability of finding a $t$-score this large or larger [ $p$ (cor)], and location in MNI space. The $x, y$, $z$ coordinates represent approximate millimeter distances from the anterior commissure (voxel 000 ), with positive values to the right, anterior, and superior directions, respectively. Formal group comparisons are presented in the rightmost columns. All these results represent larger BOLD signal in the IBS-C group. It should be noted that this represents a bigger change from the resting baseline state in the control group in ROIs where the primary response to a stimulus was a $\mathrm{BOLD}$ decrease. ROI ${ }^{0.01}$, Proportion of the region of interest where $p<0.01$ (uncorrected). ${ }^{*} p<0.0005$.

the bilateral aINS, with a suggestive trend in left dACC. They generated a significant BOLD decrease in left AMYG. There were no significant effects in control subjects but also no significant differences between the groups during sham distention.

\section{Relationship of anticipatory brain response to} visceral perception

To interpret the observed group difference in anticipatory brain activity, we combined the groups and assessed the relationship between DBS BOLD response during anticipation 
Table 4. Direct covariation of cue BOLD response with SSR negative affect

\begin{tabular}{|c|c|c|c|c|c|c|c|c|c|}
\hline & \multicolumn{3}{|c|}{ Cluster } & \multicolumn{5}{|c|}{ Voxel } \\
\hline & & $k$ & $p$ (cor) & $\mathrm{RO} \mathrm{I}^{0.01}$ & $t$ & $p$ (cor) & $x$ & $y$ & $Z$ \\
\hline \multicolumn{10}{|l|}{ Anxiety } \\
\hline \multirow[t]{2}{*}{ Dorsal brainstem } & $\mathrm{L}$ & 68 & 0.01 & $20 \%$ & 4.29 & 0.02 & 0 & -28 & -20 \\
\hline & R & 50 & 0.04 & $21 \%$ & 4.54 & 0.009 & 2 & -28 & -20 \\
\hline \multicolumn{10}{|l|}{ Anger } \\
\hline \multirow[t]{2}{*}{ Dorsal brainstem } & $\mathrm{L}$ & 67 & 0.02 & $20 \%$ & 4.55 & 0.009 & -2 & -24 & -16 \\
\hline & $\mathrm{R}$ & 28 & 0.08 & $15 \%$ & 3.83 & 0.04 & 4 & -30 & -24 \\
\hline \multirow[t]{2}{*}{ Amygdala } & L & 16 & 0.12 & $7 \%$ & 3.79 & 0.04 & -26 & 0 & -26 \\
\hline & $\mathrm{R}$ & & & & & & & & \\
\hline \multirow[t]{2}{*}{ ACC infragenual } & L & & & & & & & & \\
\hline & $\mathrm{R}$ & 22 & 0.10 & $6 \%$ & 4.01 & 0.03 & 12 & 46 & -8 \\
\hline \multicolumn{10}{|l|}{ Stress } \\
\hline \multirow[t]{2}{*}{ Dorsal brainstem } & L & 68 & 0.02 & $19 \%$ & 3.96 & 0.03 & -2 & -24 & -16 \\
\hline & $\mathrm{R}$ & 33 & 0.07 & $15 \%$ & 4.40 & 0.01 & 4 & -30 & -24 \\
\hline \multirow[t]{2}{*}{ Amygdala } & L & 20 & 0.10 & $8 \%$ & 3.35 & 0.09 & -26 & 0 & -26 \\
\hline & $\mathrm{R}$ & & & & & & & & \\
\hline \multirow[t]{2}{*}{ ACC infragenual } & L & & & & & & & & \\
\hline & $\mathrm{R}$ & 25 & 0.09 & $7 \%$ & 3.54 & 0.07 & 10 & 46 & -8 \\
\hline \multirow[t]{2}{*}{ Anterior insula } & $\mathrm{L}$ & & & & & & & & \\
\hline & R & 45 & 0.10 & $12 \%$ & 4.23 & 0.05 & 36 & 10 & 2 \\
\hline \multirow[t]{2}{*}{ Posterior insula } & L & 17 & 0.33 & $6 \%$ & 4.18 & 0.07 & -44 & -10 & 8 \\
\hline & $R$ & 67 & 0.05 & $7 \%$ & 3.43 & 0.21 & 42 & -8 & 10 \\
\hline
\end{tabular}

$\mathrm{ROI}^{0.01}$, Proportion of the ROI where $p<0.01$ (uncorrected)

and several possible explanatory measures. To dissociate the function of anticipatory BOLD decreases in the dorsal pons from those in the dorsal midbrain, the DBS voxel with maximal group differences during the cue period in the left hemisphere was selected as representative of the midbrain PAGNCF complex $(-4,-26,-12)$, and the DBS voxel with maximal group differences in the right hemisphere was selected as representative of the pontine LCC-PBN complex (4, $-30,-24)$. We first considered the potential explanatory variable of visceral perception using the rectal discomfort threshold obtained from each individual at the beginning of the fMRI session. Covariate analyses revealed that higher discomfort thresholds (e.g., less sensitivity) were associated with lower BOLD cue activity in the voxels selected for maximal group differences in both LCC-PBN $(t=2.55 ; p=0.009)$, and PAG-NCF $(t=2.08 ; p=0.02)$. However, these effects were neither strong nor spatially extensive enough to retain significance after volume-correction for the entire DBS.

\section{Relationship of anticipatory brain response to affective state}

The relationship of the brainstem anticipatory response to differences in affective state was assessed using the SSR selfratings. The selected voxels had higher anticipatory BOLD signal to the extent that self-rated negative affect was higher in both LCC-PBN [ $t=4.30$ (anxiety), 3.83 (anger), 4.40 (stress); all $p<0.0005]$ and PAG-NCF $[t=3.43$ (anxiety), 3.39 (anger), 3.52 (stress); all $p=0.001$ ]. Table 4 indicates that all three self-ratings remained significantly correlated with the BOLD signal during cued expectation of pain after volumecorrection for the full DBS. Thus, negative mood was associated with less of the anticipatory BOLD decrease found in healthy controls but not IBS-C patients as a group. Figure 3 depicts sagittal and axial views of the positive correlation between negative affect (anxiety, anger, and stress) and the anticipatory BOLD signal in DBS, with crosshairs centered on the voxel of peak effect in the right hemisphere, which was in LCC-PBN for all three scales. In the transaxial view and in
Table 4, the data suggest that activity in the left lateral AMYG had a similar relationship with anger and stress. Anger and stress were also associated with less anticipatory BOLD decrease in the right iACC, and stress was associated with a smaller BOLD decrease in both anterior and posterior INS (Table 4).

\section{Relationship of anticipatory brainstem response to the brain response to distention}

Covariate analyses assessed the relationship of the cue-elicited BOLD signal in LCC-PBN (voxel $4,-30,-24$ ) and PAG-NCF (voxel $4,-26,-12$ ) to the brain response during the $45 \mathrm{mmHg}$ distention. The PAG-NCF cue response was not related to the response to $45 \mathrm{~mm}$ distention in any ROI. In contrast, as depicted in Figure 4, anticipatory deactivation of LCC-PBN (blue circle) was associated with greater activation during subsequent 45 $\mathrm{mmHg}$ distention in bilateral rostral ACC, with extension both below the genu (13\% of L iACC: spatial extent, $p=0.05$; peak $t=$ $4.15 ; p=0.02 ; 22 \%$ of R iACC: spatial extent, $p=0.02$; peak $t=$ 4.04; $p=0.03$ ), and above (18\% of L sACC: spatial extent, $p=$ 0.007 ; peak $t=4.03 ; p=0.05$ ).

Figure 4 also illustrates that anticipatory deactivation of LCC$\mathrm{PBN}$ was associated with greater activation during subsequent 45 $\mathrm{mmHg}$ distention in the right, but not left, lateral OFC. The peak association of right OFC to the LCC-PBN cue signal at voxel 36, $42,-10$ yielded a t-score of 4.57 (volume corrected, $p=0.04$ ). The voxel was in BA 47, part of a cluster of 338 voxels representing $24 \%$ of the ROI (volume corrected spatial extent, $p<0.001$ ). The homologous comparison (thresholded at $p<0.01$ ) in the left lateral OFC yielded only one voxel.

To further assess the specificity of the LCC-PBN anticipatory deactivation for greater activation of rostral ACC and right lateral OFC during distention, we conducted an additional analysis of the relationship of the cue-elicited BOLD signal in the left amygdala (voxel $-22,-4,-24$ ) to the brain response during the 45 $\mathrm{mmHg}$ distention. There was no evidence for any relationship analogous to the one reported for the LCC-PBN and depicted in Figure 4 . The only ROI where anticipatory deactivation of left amygdala was associated with greater activation during subsequent $45 \mathrm{mmHg}$ distention was the left amygdala itself (peak, $p=$ 0.02 ), and this effect was very focal, covering only $3 \%$ of the left amygdala.

\section{Discussion}

The brain must evaluate tissue damage during pain and generate behavioral responses but must also be able to inhibit response in favor of competing goals. Recent studies have associated BOLD decreases with decreased neural firing (Logothetis et al., 2001; Shmuel et al., 2002). Limbic downregulation during pain anticipation has been suggested as a cognitive coping strategy (Petrovic et al., 2004). The current results demonstrate that during certain expectation of experimental abdominal/pelvic discomfort, female IBS-C patients are more anxious and less able than healthy controls to downregulate activity within the CNS network activated by potentially aversive interoceptive stimuli, especially within DBS. During aversive stimulation, they generate more activity than controls in DBS and dACC, and to the extent that preparatory inhibition of the LCC-PBN region fails, patients do not activate a well-described right lateral OFC-sACC cortical coping network. These findings are consistent with our recently proposed model conceptualizing CNS abnormalities in IBS as ineffective cortico-limbic-pontine inhibition of visceral afferent 
input within the homeostatic afferent processing network (Mayer et al., 2006).

\section{Altered brain responses during expectation of an aversive visceral stimulus in patients}

Healthy controls, but not patients, showed extensive BOLD decreases during expectation of visceral discomfort in key regions of a brain network, which is consistently activated during perturbation of homeostasis and mediates the sensory and affective/motivational aspects of homeostatic emotions, including visceral pain (Mayer et al., 2006). The only major component of the afferent processing network that was not an a priori ROI in this study was the thalamus. Post hoc analyses showed that healthy controls also deactivated the thalamus extensively, whereas patients did not.

However, as with the cue-response of several other network structures, formal group differences in the thalamus did not attain significance, suggesting that some patients may also decrease network activity when anticipating discomfort. Individual analyses revealed cue-related BOLD decrease of the LCC-PBN in all healthy subjects, but only $36 \%$ of IBS-C patients. Subsequent activity in the aINS during sham distention (Table 3) demonstrates that in the absence of a stimulus, anticipation in IBS patients only, perhaps because of higher anxiety, can increase firing within interoceptive cortex. This may indicate continued ascending arousal and/or lack of descending prefrontal inhibitory input.

During cued expectation, the DBS was the primary region showing greater activation in IBS-C patients than in healthy controls. Although our study cannot differentiate individual pontine nuclei, we speculate that this greater activation probably involves the LCC, a small cylindrical structure abutting the PBN, which provides most forebrain noradrenergic inputs and is implicated in arousal (AstonJones and Cohen, 2005).

A recent study reported that PAG activation during anticipated pain was associated with higher pain ratings and greater pINS activation in healthy adults (Fairhurst et al., 2007). However, no anticipatory brainstem activation reached the $p<0.01$ level, and BOLD decreases were not assessed, so it is unclear whether they were present.

Alterations in preparatory responses are correlated with measures of negative affect and with brain responses to the delivered pain stimulus

Elevated symptoms of anxiety in IBS patients (Mayer et al., 2001) may reflect an intrinsic pathophysiological component in this and other functional pain disorders. Our patients had more negative affect during scanning than control subjects, and these ratings correlated with brainstem activity during expectation. Correlation of cue BOLD signal with negative affect is consistent with LCC arousal.

The current results during visceral distention replicate previous studies of brain activity during pain both through finding activity increases in the afferent processing network structures of INS, dACC, and DBS (Mayer et al., 2006) and activity decreases in the rostral ACC (Porro et al., 1998, 2004) and amygdala (Derbyshire et al., 1997; Baciu et al., 1999; Petrovic et al., 1999; Berman et al., 2002a; Petrovic and Ingvar, 2002).

Patients had more BOLD activation in LCC-PBN and dACC than healthy women. Previous imaging studies compar- 
ing IBS patients to healthy controls during rectal distention have also reported greater activation of the dACC (Mertz et al., 2000; Naliboff et al., 2001; Mayer et al., 2005;). It remains to be determined whether these differences are best interpreted as decreased inhibitory corticolimbic antinociception/coping mechanisms (Wilder-Smith et al., 2004; Mayer et al., 2005; Song et al., 2006) or also reflect increased ascending afferent information (Verne et al., 2003). To address this issue, we looked for correlations between the observed brain responses during expectation (in the absence of any peripheral input) and the response to the actual stimulus.

When the cue was not followed by actual distention (sham condition), the voxels where the anticipatory LCC-PBN BOLD signal remained most directly correlated with activity over the next $15 \mathrm{~s}$ were in basomedial thalamus $(t=4.6)$ and LCC-PBN itself $(t=4.5)$, supporting the notion that variation in anticipatory LCC-PBN activity represents modulation of background noise levels in the circuit for detection of visceral afferent information (Saleh and Cechetto, 1994). In contrast, when a $45 \mathrm{mmHg}$ distention occurred, high LCC-PBN activity during the cue was associated with less activation of sACC and right OFC/ventrolateral PFC; cortical regions associated with antinociception through opiate-mediated placebo effects (Petrovic and Ingvar, 2002; Eisenberger et al., 2003; Lieberman et al., 2004; Petrovic et al., 2005; Bingel et al., 2006) and analgesic effects of perceived control over pain (Wiech et al., 2006). During actual distention, the cue signal was unrelated to the level of activation in areas most reliably activated during pain (dACC, INS), consistent with animal studies where noradrenergic modulation of PBN changed the signal-to-noise ratio but did not directly alter stimulus-specific firing elicited by moderate visceral stimulation (Saleh and Cechetto, 1994).

\section{Possible pathophysiological model of altered noradrenergic modulation during expectation of pain}

Recent studies suggest that the LCC orchestrates more flexible optimization of performance through mixing stimulus-driven phasic activity that is an important neural substrate of focused attention with tonic activity that encourages nonspecific fast responding and behavioral switching (Aston-Jones and Cohen, 2005). This model suggests two strategies that might be applied during expectation of discomfort. Increasing phasic responsiveness and decreasing tonic (nonspecific) firing of the LCC could optimize the signal-to-noise ratio, and the accuracy of perceptual detection of weak or attended stimuli, while decreasing responsiveness to unattended stimuli. Alternatively, increasing tonic firing of the LCC and thus the firing rate in upstream thalamus closer to the threshold for pain detection would shorten reaction time nonspecifically to any additional source of arousal. Because speeded response to actual threats would be accompanied by increased false alarms, this strategy for fast detection of potential threats constitutes a bias to interpret network activity as threatening.

Use of this strategy by IBS patients is consistent with previous reports demonstrating enhanced central reactivity (Berman et al., 2002b; Naliboff et al., 2005), and an intriguing study where IBS patients made more false-positive errors in recognizing emotionally negative words than either healthy subjects or depressed patients (Gomborone et al., 1993). We propose that during expectation of pain, IBS patients generate higher levels of tonic noradrenergic activity, producing a bias toward interpretation of network activity as pain (speed over accuracy), and are inefficient at reducing such activity when discrimination of nonpainful stimulation should be maximized, or pain should be inhibited.

\section{Limitations of study}

Findings obtained in moderate to severe female IBS-C patients may not generalize to other patient populations. We did not image the rostroventral medulla, and limited spatial resolution prevented potentially important discriminations within closely interconnected brainstem networks. Future imaging studies of anticipation and pain could achieve better resolution through using smaller voxels and coronal slices parallel to the brainstem.

Substantial group differences in negative affect make it difficult to disentangle these effects from other group differences. In particular, IBS patients showed higher anxiety ratings, and two had significantly elevated anxiety. However, unlike the cue response, brain response to the uncomfortable stimulus itself was not related to negative affect or discomfort threshold, suggesting that negative mood states inhibit the preparatory deactivation that is our focus but do not strongly alter the brain response to a moderate stimulus.

\section{Summary}

We provide evidence for a relationship between the altered brain and perceptual responses to visceral stimuli reported in IBS and alterations in brain response to expectation of such stimuli. During anticipation of visceral pain, healthy subjects, but not IBS patients, downregulate homeostatic afferent processing network activity. Downregulation is maximal within dorsal pons (LCC-PBN) and associated both with higher rectal distention thresholds in healthy subjects (improved discrimination of physiological from aversive stimuli) and with activation of an antinociceptive network in right lateral OFC and sACC during delivered pain. Anticipatory downregulation is inhibited by negative emotions (stress, anxiety, anger), and these are higher in IBS patients. Negative emotions support a competing strategy of tonic LCC arousal to disinhibit behavioral response, consistent with IBS patients having greater DBS and AACC activation during actual distention and false positive detection of interoceptive information (INS activity) during sham distention.

The proposed model of altered noradrenergic regulation in IBS requires confirmation in future studies. However, altered LCC function has been proposed previously as an important pathophysiological mechanism in functional pelvic pain disorders (Valentino et al., 1999).

\section{References}

Asmundson GJ, Norton PJ, Norton GR (1999) Beyond pain: the role of fear and avoidance in chronicity. Clin Psych Rev 19:97-119.

Aston-Jones G, Cohen D (2005) An integrative theory of locus coeruleusnorepinephrine function: adaptive gain and optimal performance. Annu Rev Neurosci 28:403-450.

Baciu MV, Bonaz BL, Papillon E, Bost RA, Le Bas JF, Fournet J, Segebarth CM (1999) Central processing of rectal pain: a functional MR imaging study. Am J Neuroradiol 20:1920-1924.

Berman SM, Suyenobu B, Gordon W, Mandelkern M, Naliboff BD, Mayer E (2002a) Evidence for antinociceptive deactivation of the amygdala in functional GI disorders. Gastroenterology 122:A313.

Berman SM, Naliboff BD, Chang L, FitzGerald L, Antolin T, Camplone A, Mayer EA (2002b) Enhanced preattentive central nervous system reactivity in irritable bowel syndrome. Am J Gastroenterol 97:2791-2797. 
Bingel U, Lorenz J, Schoell E, Weiller C, Buchel C (2006) Mechanisms of placebo analgesia: rACC recruitment of a subcortical antinociceptive network. Pain 120:8-15.

Bjelland I, Dahl AA, Haug TT, Neckelmann D (2002) The validity of the Hospital Anxiety and Depression Scale. An updated literature review. J Psychosom Res 52:69-77.

Bonaz B, Baciu M, Papillon E, Bost R, Gueddah N, Le Bas JF, Fournet J, Segebarth C (2002) Central processing of rectal pain in patients with irritable bowel syndrome: an fMRI study. Am J Gastroenterol 97:654-661.

Bushnell MC, Duncan GH, Dubner R, Jones RL, Maixner W (1985) Attentional influences on noxious and innocuous cutaneous heat detection in humans and monkeys. J Neurosci 5:1103-1110.

Coull JT (1998) Neural correlates of attention and arousal: insights from electrophysiology, functional neuroimaging and psychopharmacology. Prog Neurobiol 55:343-361.

Derbyshire SW, Jones AK, Gyulai F, Clark S, Townsend D, Firestone LL (1997) Pain processing during three levels of noxious stimulation produces differential patterns of central activity. Pain 73:431-445.

Diatchenko L, Nackley AG, Slade GD, Fillingim RB, Maixner W (2006) Idiopathic pain disorders-pathways of vulnerability. Pain 123:226-230.

Dickhaus B, Mayer EA, Firooz N, Stains J, Conde F, Olivas TI, Fass R, Chang L, Mayer M, Naliboff BD (2003) Irritable bowel syndrome patients show enhanced modulation of visceral perception by auditory stress. Am J Gastroenterol 98:135-143.

Drossman DA (2006) The functional gastrointestinal disorders and the Rome III process. Gastroenterology 130:1377-1390.

Dunckley P, Wise RG, Fairhurst M, Hobden P, Aziz Q, Chang L, Tracey I (2005) A comparison of visceral and somatic pain processing in the human brainstem using functional magnetic resonance imaging. J Neurosci 25:7333-7341.

Eisenberger NI, Lieberman MD, Williams KD (2003) Does rejection hurt? An fMRI study of social exclusion. Science 302:290-292.

Fairhurst M, Wiech K, Dunckley P, Tracey I (2007) Anticipatory brainstem activity predicts neural processing of pain in humans. Pain 128:101-110.

Gomborone JE, Dewsnap PA, Libby GW, Farthing MJ (1993) Selective affective biasing in recognition memory in the irritable bowel syndrome. Gut 34:1230-1233.

Goubert L, Crombez G, Van Damme S (2004) The role of neuroticism, pain catastrophizing and pain-related fear in vigilance to pain: a structural equations approach. Pain 107:234-241.

Gracely RH, McGrath P, Dubner R (1978) Ratio scales of sensory and affective verbal pain descriptors. Pain 5:5-18.

Gracely RH, Geisser ME, Giesecke T, Grant MA, Petzke F, Williams DA, Clauw DJ (2004) Pain catastrophizing and neural responses to pain among persons with fibromyalgia. Brain 127:835-843.

Hsieh JC, Stone-Elander S, Ingvar M (1999) Anticipatory coping of pain expressed in the human anterior cingulate cortex: a positron emission tomography study. Neurosci Lett 262:61-64.

Keogh E, Thompson T, Hannent I (2003) Selective attentional bias, conscious awareness and the fear of pain. Pain 104:85-91.

Labus JS, Mayer EA, Chang L, Bolus R, Naliboff BD (2007) The central role of gastrointestinal-specific anxiety in irritable bowel syndrome: further validation of the Visceral Sensitivity Index. Psychosomatic Med 69:89-98.

Lembo T, Munakata J, Mertz H, Niazi N, Kodner A, Nikas V, Mayer E (1994) Evidence for the hypersensitivity of lumbar splanchnic afferents in irritable bowel syndrome. Gastroenterology 107:1686-1696.

Leuchter AF, Cook IA, Witte EA, Morgan M, Abrams M (2002) Changes in brain function of depressed subjects during treatment with placebo. Am J Psychiatry 159:122-129.

Lieberman MD (2005) Principles, processes, and puzzles of social cognition: an introduction for the special issue on social cognitive neuroscience. NeuroImage 28:745-756.

Lieberman MD, Jarcho JM, Berman S, Naliboff BD, Suyenobu BY, Mandelkern M, Mayer EA (2004) The neural correlates of placebo effects: a disruption account. NeuroImage 22:447-455.

Logothetis NK, Pauls J, Augath M, Trinath T, Oeltermann A (2001) Neurophysiological investigation of the basis of the fMRI signal. Nature 412:150-157.
Mayer EA, Craske MG, Naliboff BD (2001) Depression, anxiety and the gastrointestinal system. J Clin Psychiatry 62:28-36.

Mayer EA, Berman S, Suyenobu B, Labus J, Mandelkern MA, Naliboff BD, Chang L (2005) Differences in brain responses to visceral pain between patients with irritable bowel syndrome and ulcerative colitis. Pain 115:398-409.

Mayer EA, Naliboff BD, Craig AD (2006) Neuroimaging of the brain-gut axis: from basic understanding to treatment of functional GI disorders. Gastroenterology 131:1925-1942.

Mertz H, Walsh JH, Sytnik B, Mayer EA (1995) The effect of octreotide on human gastric compliance and sensory perception. Neurogastroenterol Motil 7:175-185.

Mertz H, Morgan V, Tanner G, Pickens D, Price R, Shyr Y, Kessler R (2000) Regional cerebral activation in irritable bowel syndrome and control subjects with painful and nonpainful rectal distension. Gastroenterology 118:842-848.

Naliboff BD, Benton D, Solomon GF, Morley JE, Fahey JL, Bloom ET, Makinodan T, Gilmore SL (1991) Immunological changes in young and old adults during brief laboratory stress. Psychosomatic Med 53:121-132.

Naliboff BD, Munakata J, Fullerton S, Gracely RH, Kodner A, Harraf F, Mayer EA (1997) Evidence for two distinct perceptual alterations in irritable bowel syndrome. Gut 41:505-512.

Naliboff BD, Derbyshire SWG, Munakata J, Berman S, Mandelkern M, Chang L, Mayer EA (2001) Cerebral activation in irritable bowel syndrome patients and control subjects during rectosigmoid stimulation. Psychosomatic Med 63:365-375.

Naliboff BD, Waters A, Labus J, Murphy S, Mayer EA, Ornitz EM (2005) Increased anxiety potentiated startle in irritable bowel syndrome (IBS): evidence for central hyperexcitability. Gastroenterology 128:A-340.

Petrovic P, Ingvar M (2002) Imaging cognitive modulation of pain processing. Pain 95:1-5.

Petrovic P, Ingvar M, Stone-Elander S, Petersson KM, Hansson P (1999) A PET activation study of dynamic mechanical allodynia in patients with mononeuropathy. Pain 83:459-470.

Petrovic P, Carlsson K, Petersson KM, Hansson P, Ingvar M (2001) Context dependent amygdala deactivation during pain. NeuroImage 13:S457.

Petrovic P, Kalso E, Petersson KM, Ingvar M (2002) Placebo and opioid analgesia-imaging a shared neuronal network. Science 295:1737-1740.

Petrovic P, Carlsson K, Petersson KM, Hansson P, Ingvar M (2004) Context-dependent deactivation of the amygdala during pain. J Cogn Neurosci 16:1289-1301.

Petrovic P, Dietrich T, Fransson P, Andersson J, Carlsson K, Ingvar M (2005) Placebo in emotional processing-induced expectations of anxiety relief activate a generalized modulatory network. Neuron 46:957-969.

Porro CA, Cettolo V, Francescato MP, Baraldi P (1998) Temporal and intensity coding of pain in human cortex. J Neurophysiol 80:3312-3320.

Porro CA, Baraldi P, Pagnoni G, Serafini M, Facchin P, Maieron M, Nichelli P (2002) Does anticipation of pain affect cortical nociceptive systems? J Neurosci 22:3206-3214.

Porro CA, Cettolo V, Francescato MP, Baraldi P (2003) Functional activity mapping of the mesial hemispheric wall during anticipation of pain. NeuroImage 19:1738-1747.

Porro CA, Lui F, Facchin P, Maieron M, Baraldi P (2004) Percept-related activity in the human somatosensory system: functional magnetic resonance imaging studies. Magn Reson Imag 22:1539-1548.

Saleh TM, Cechetto DF (1994) Neurotransmitters in the parabrachial nucleus mediating visceral input to the thalamus in rats. Am J Physiol 266:R1287-R1296.

Sawamoto N, Honda M, Okada T, Hanakawa T, Kanda M, Fukuyama H, Konishi J, Shibasaki H (2000) Expectation of pain enhances responses to nonpainful somatosensory stimulation in the anterior cingulate cortex and parietal operculum/posterior insula: an eventrelated functional magnetic resonance imaging study. J Neurosci 20:7438-7445. 
Shmuel A, Yacoub E, Pfeuffer J, Van de Moortele PF, Adriany G, Hu X, Ugurbil K (2002) Sustained negative BOLD, blood flow and oxygen consumption response and its coupling to the positive response in the human brain. Neuron 36:1195-1210.

Simpson Jr JR, Drevets WC, Snyder AZ, Gusnard DA, Raichle ME (2001) Emotion-induced changes in human medial prefrontal cortex II. During anticipatory anxiety. Proc Natl Acad Sci USA 98:688-693.

Song GH, Venkatraman V, Ho KY, Chee MW, Yeoh KG, Wilder-Smith CH (2006) Cortical effects of anticipation and endogenous modulation of visceral pain assessed by functional brain MRI in irritable bowel syndrome patients and healthy controls. Pain 126:79-90.

Valentino RJ, Miselis RR, Pavcovich LA (1999) Pontine regulation of pelvic viscera: pharmacological target for pelvic visceral dysfunction. Trends Pharmacol Sci 20:253-260.

Verne GN, Himes NC, Robinson ME, Gopinath KS, Briggs RW, Crosson B,
Price DD (2003) Central representation of visceral and cutaneous hypersensitivity in the irritable bowel syndrome. Pain 103:99-110.

Wessely S, Nimnuan C, Sharpe M (1999) Functional somatic syndromes: one or many? Lancet 354:936-939.

Whitehead WE, Palsson OS (1998) Is rectal pain sensitivity a biological marker for irritable bowel syndrome: psychological influences on pain perception. Gastroenterology 115:1263-1271.

Wiech K, Kalisch R, Weiskopf N, Pleger B, Stephan KE, Dolan RJ (2006) Anterolateral prefrontal cortex mediates the analgesic effect of expected and perceived control over pain. J Neurosci 26:11501-11509.

Wilder-Smith CH, Schindler D, Lovblad K, Redmond SM, Nirkko A (2004) Brain functional magnetic resonance imaging of rectal pain and activation of endogenous inhibitory mechanisms in irritable bowel syndrome patient subgroups and healthy controls. Gut 53:1595-1601. 